Article

\title{
Engineering Aspheric Liquid Crystal Lenses by Using the Transmission Electrode Technique
}

\author{
José Francisco Algorri ${ }^{1, *} \mathbb{C}$, Dimitrios C. Zografopoulos ${ }^{2} \mathbb{(}$, Luis Rodríguez-Cobo $^{3}{ }^{(\mathbb{C}}$, \\ José Manuel Sánchez-Pena ${ }^{4}$ (D) and José Miguel López-Higuera ${ }^{1,3,5}$ (D) \\ 1 Photonics Engineering Group, University of Cantabria, 39005 Santander, Spain; \\ miguel.lopezhiguera@unican.es \\ 2 Consiglio Nazionale delle Ricerche, Istituto per la Microelettronica e Microsistemi, 00133 Rome, Italy; \\ dimitrios.zografopoulos@artov.imm.cnr.it \\ 3 CIBER-bbn, Instituto de Salud Carlos III, 28029 Madrid, Spain; luis.rodriguez@unican.es \\ 4 GDAF-UC3M, Displays and Photonics Applications Group, Department of Electronic Technology, \\ Carlos III University of Madrid, Leganés, 28911 Madrid, Spain; jmpena@ing.uc3m.es \\ 5 Instituto de Investigación Sanitaria Valdecilla (IDIVAL), 39011 Santander, Spain \\ * Correspondence: algorrijf@unican.es
}

Received: 26 August 2020; Accepted: 16 September 2020; Published: 18 September 2020

\begin{abstract}
The transmission electrode technique has been recently proposed as a versatile method to obtain various types of liquid-crystal (LC) lenses. In this work, an equivalent electric circuit and new analytical expressions based on this technique are developed. In addition, novel electrode shapes are proposed in order to generate different phase profiles. The analytical expressions depend on manufacturing parameters that have been optimized by using the least squares method. Thanks to the proposed design equations and the associated optimization, the feasibility of engineering any kind of aspheric LC lenses is demonstrated, which is key to obtain aberration-free lenses. The results are compared to numerical simulations validating the proposed equations. This novel technique, in combination with the proposed design equations, opens a new path for the design and fabrication of LC lenses and even other types of adaptive-focus lenses based on voltage control.
\end{abstract}

Keywords: adaptive-focus lenses; liquid-crystal lenses; phase modulation

\section{Introduction}

Adaptive-focus lenses are capable of tuning their focal length by using some kind of external stimulus. In order to obtain optical power tunability, either the curvature of their interface or the spatial profile of the constituent material's refractive index need to be modified. Many techniques have been thus far used to obtain the aforementioned effects. They can be grouped in three main categories: sliding, shape-changing, and refractive index-controlled lenses. The first group is also known as Alvarez lenses. They are now gaining attention for miniature adaptive lenses [1] and high-speed focusing [2]. These lenses show fast switching in combination with broad optical power range. The shape-changing technique embraces several structures, for example, based on elastic membranes [3-6], ferrofluidics [7-9], soft electroactive actuators [10], and the electrowetting and dielectrophoretic effect $[11,12]$. For this reason, the characteristics vary widely depending upon the nature of the surface or the physical effect. In general, the main disadvantages associated with this approach are the usually high operating voltage, the increased thickness necessary for large aperture lenses (in order to avoid the capillary effect) and detrimental reflection and scattering due to multiple interfaces. Finally, adaptive-focus lenses based on the gradual variation of the refractive index do not need curved surfaces. Instead, such lenses are made by using a material that exhibits a spatial 
gradient of its refractive index. One of the most common materials for this purpose is liquid crystals (LC). Many of the topologies proposed for LC lenses are based on generating a gradual voltage drop across the lens surface. This voltage profile produces a refractive index gradient in the LC layer by means of the LC electro-optic effect, thus mimicking the optical behavior of a conventional lens with the great advantage of volume/weight reduction, and tunable focal distance.

Despite the fact that LC lenses were first reported 40 years ago $[13,14]$, they still remain an active field of research. Nowadays, there is huge research effort directed to devising structures capable of generating LC-tunable counterparts of classic optical components. Some examples are large lenses [15,16], multi-focal [17,18], high fill-factor [19] and frequency-controlled [20] microlenses, tunable zooming [21], beam steering [22,23], beam splitters [24], diffraction gratings [25], aberration correctors [26,27] astronomy [28], 3D vision applications [29-32], optical filters [33], optical switches [34], micro-axicon arrays [35], lensacons and logarithmic axicons [36,37], and optical vortices [38-41]. Potential applications in practice span the entire range of classic optical components, albeit with the advantages previously mentioned. An extensive overview and more information can be found in some of the recent reviews on this topic, for example, fast-response time LC microlenses [42], LC microlenses for autostereoscopic displays [43], design and fabrication [44], LC contact lenses for the correction of presbyopia [45] or recent developments [46]. Also, a more general review of the different topologies can be found in Reference [47].

Two of the most important approaches for the fabrication of adaptive-focus LC lenses are the patterned $[48,49]$ and the modal [50] electrode techniques. The first category comprises multielectrode lenses, which are one of the most versatile solutions due to the fully configurable phase profiles. The main problem is the complex fabrication process (usually involving several layers of electrodes) and the complex voltage control (each electrode requires a distinct voltage). On the other hand, the modal control overpasses previous shortcomings by using a high resistivity layer that distributes homogeneously the voltage across the active area [51]. In this case, only one or two low voltage sources are required but fabrication is still an issue given the difficulty to obtain homogenous high resistivity layers (evaporation of thin layers over large apertures are necessary). In order to solve all of these problems a novel technique has been recently proposed, the transmission electrode technique. It combines all the advantages of previous structures but fabrication and voltage control are much simpler and straightforward. By exploiting this technique, we have recently demonstrated axicon and spherical lenses [52], cylindrical and Powell lenses [53] and mutioptical systems [54].

In this work, this technique is analytically studied in order to engineer any kind of aspheric LC lenses, which is crucial in order to obtain aberration-free lenses. We demonstrate that this versatile technique allows the design of the spatial phase profile by changing only two parameters of the electrode. Thanks to the proposed design equations, it is possible to adjust the profiles to different analytical mathematical curves, for example, parabolas, ellipses, and hyperbolas. The results are compared with numerical simulations showing excellent agreement. This novel technique, in combination with the proposed design equations, opens a new path to fabricate LC lenses and even other types of adaptive-focus lenses based on voltage control.

\section{Analytical Study of the Transmission Electrode Technique}

The transmission electrode technique is based on two main elements. The first one is a central transmission electrode that distributes the voltage between two voltage sources, as in Figure 1a,b. A low resistivity material can be used, a straightforward solution being commercial ITO-on-glass substrates. Despite the low resistivity, the total resistance between the contacts is high $(\mathrm{k} \Omega-\mathrm{M} \Omega)$ due to the high aspect ratio of the transmission electrode (length/width). In the first example, shown in Figure $1 \mathrm{a}$, a voltage divider is obtained. In this configuration, the central voltage, $V_{C}$, can be controlled by $V_{2}$ [52]. In the second example, Figure $1 \mathrm{~b}$, when the applied voltages have fixed phase shifts $V_{1}=A_{1}<0^{\circ} ; V_{2}=A_{2}<180^{\circ}$, the voltage goes from $A_{1}$ to $-A_{2}$, crossing in the middle by zero when the two amplitudes match $\left(A_{1}=A_{2}\right)$. Moreover, if necessary the central voltage can be 
offset from zero by using some special signals (see, for instance, Reference [54])). The profile of the voltage distribution between the lateral and central points $x_{1}$ and $x_{2}$, respectively, can be designed by modifying the transmission electrode distributed resistance between these two points. Specifically, by modifying the shape of the electrode the voltage profile is also modified accordingly. As it will be demonstrated in the following sections, the optical phase profiles resulting from such voltage profiles can approach linear, parabolic, elliptical and hyperbolic profiles, enabling the generation of all kinds of aspheric lenses.

The second element consists of a series of parallel or concentric electrode stubs evenly arranged, as in Figure 1c,d, which show two standard configurations corresponding to spherical and cylindrical lenses. These electrodes distribute the voltage across the active area, behaving like the high resistivity layer of modal devices but with the advantage of being made by a simple ITO lithographic process and having low resistivity. The gaps between electrodes have to be as short as possible to avoid abrupt phase steps. This discontinuity effect is proportional to the gap/LC cell thickness aspect ratio [52]. As the gap can be brought down towards micrometric resolution using standard photolithography, the phase-discontinuity effect is negligible for LC cell thicknesses above $10 \mu \mathrm{m}$ (i.e., roughly for aspect ratios above 1:10), which provides ample space for device engineering.

The combination of these two elements allows a simple study. By estimating the voltage in the central transmission electrode, the voltage across the active area can be inferred. In the next section, a simple Equivalent Electrical Circuit (EEC) is proposed in order to calculate the voltage at each point of the transmission electrode. Then, the equations for different electrode shapes are elaborated.
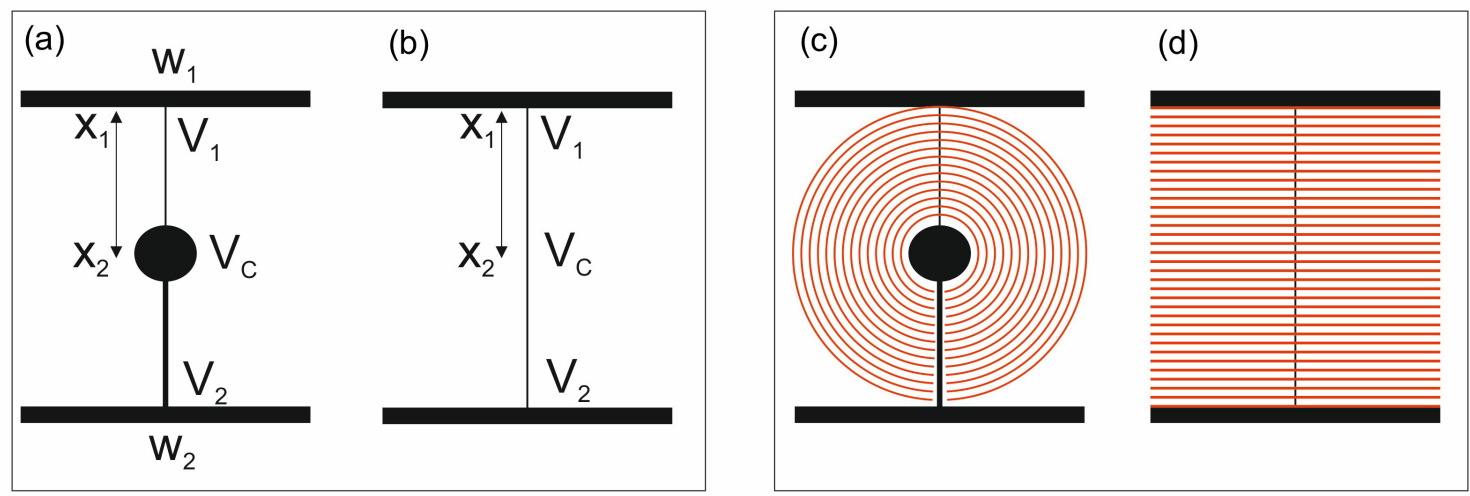

Figure 1. Schematic diagram of the transmission electrode technique. Detail of the transmission electrode (in black) for (a) voltage divider configuration and (b) orthogonal configuration. Detail of the (c) concentric and (d) parallel electrodes stubs (in red) that distribute the voltage over the active area. Note in (c) that the stubs do not touch the bottom part of the transmission electrode.

\subsection{Voltage Distribution}

As commented before, the parallel/concentric electrodes act like the high resistivity layer of a modal lens. Some years ago, some of the authors proposed a general EEC for modal/hole patterned lenses, described in Figure 2a $[55,56]$. The model resembles that of a transmission line with the inclusion of a coplanar capacitor $\left(C_{1}\right)$ and a loss resistance $(G)$. In the modal topology, the distributed resistors are $R_{i}=R_{\mathrm{sq}}$, (sheet resistance of the high resistivity layer). Alternatively, in a hole-patterned structure $R_{i}=R_{\mathrm{LC}}$, modelling the LC dielectric losses in the lens surface. In the case of the transmission electrode technique, this circuit is simplified considerably, as shown in Figure 2b. Unlike the case of modal control, $R$ is very low so the rest of the components can be neglected. Thanks to this, reactive components, and consequently the temporal dependence, are dropped. There is no need to use differential equations and the system can be modeled by considering only the distributed resistance of the transmission electrode, as depicted in Figure $2 b$. 
This model is always valid when commercial ITO-on-glass substrates are used (to be in the modal control regime sheet resistances in the order of $\mathrm{M} \Omega$ would be necessary). In order to have low current between electrodes, for example, in the order of $\mu \mathrm{A}$, the resistance between contacts has to be in the order of several $\mathrm{k} \Omega$. For a fixed-width electrode, the resistance is equal to the sheet resistance (see also Section 2.2) multiplied by the aspect ratio $L / w$, where $L$ and $w$ is the electrode length and width, respectively. Considering a diameter of the active are of the proposed devices equal to $1 \mathrm{~cm}$, and transmission electrode width of $10 \mu \mathrm{m}$, the aspect ratio is about 1000. The typical commercial ITO-on-glass sheet resistance ranges from 4.5 to $500 \Omega /$ sq, corresponding to a thickness ranging from 310 to $15 \mathrm{~nm}$ respectively. This means that any typical commercial ITO-on-glass would be in principle valid for the proposed technique. Despite this, the thicker the electrode the higher the current, which could produce self-heating and losses. On the other hand, the lower the thickness the harder the fabrication due to, for instance, the difficulty to control the acid attack of thin ITO layers.
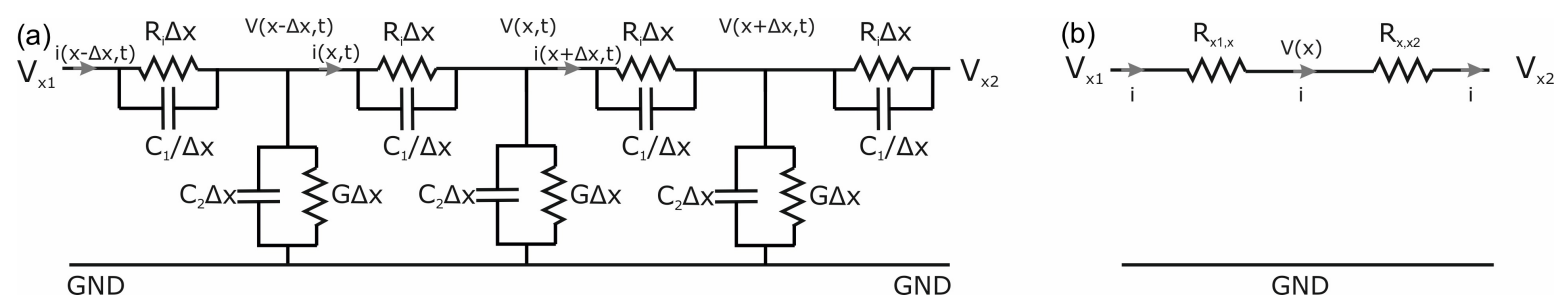

Figure 2. Equivalent electrical circuit for (a) liquid crystal (LC) lenses with modal and hole patterned configuration $[55,56]$ and (b) the transmission electrode configuration.

Specifically, the voltage $V(x)$ at a given position $x$ along the transmission electrode can be calculated in a straightforward manner employing Ohm's law in the voltage divider circuit of Figure $2 b$, with reference to the layout of Figure $1 \mathrm{~b}$, where $V_{x 1}=V_{1}$ and $V_{x 2}=V_{c}$. The voltage $V(x)$ is given by

$$
V(x)=V_{1}+i R(x)
$$

where $R(x)$ the resistance in the segment of the electrode from $x=0$ up to $x$. The current is calculated as

$$
i=\frac{\Delta V}{R_{x 1, x 2}}=\frac{\Delta V}{R_{0, x_{c}}}=\frac{V_{c}-V_{1}}{R_{t}}
$$

where $R_{t}$ is the total resistance of the electrode from $x=0$ to $x=x_{c}$. The voltage $V(x)$ is then equal to

$$
V(x)=V_{1}+\frac{V_{c}-V_{1}}{R_{t}} R(x),
$$

where $R(x)$ is calculated by

$$
R(x)=\int_{x_{1}}^{x} d R=\int_{x_{1}}^{x} \frac{\rho d x}{w(x) t}=R_{\mathrm{sq}} \int_{x_{1}}^{x} \frac{d x}{w(x)}
$$

where $\rho$ is the resistivity $t$ the thickness, $R_{\mathrm{sq}}=\rho / t$ is the sheet resistance, and $w(x)$ is the (generally) variable width of the ITO transmission electrode. By controlling $w(x)$ in the design of the photolithographic mask, different resistance and, hence, voltage profiles can be obtained, as studied in the next sections for three particular electrode shapes.

\subsection{Rectangular Electrode}

The first example is the simplest one. By using rectangular ITO electrodes, as in the structures shown in Figure 1a,b, a cuboid resistance is obtained, shown in Figure 3. The electrode has fixed width $w(x)=w$, thickness $t$, and length $L=x_{2}-x_{1}$. According to Equation (4), the resistance 
$R(x)=R_{\mathrm{sq}} x / w$ and the total electrode resistance equals $R_{t}=R_{\mathrm{sq}} L / w$. Therefore, the voltage $V(x)$ is calculated according to Equation (3)

$$
V(x)=V_{1}+\frac{V_{c}-V_{1}}{L} x .
$$

This results in a linear equation where the voltage distribution is proportional to the position $x$.

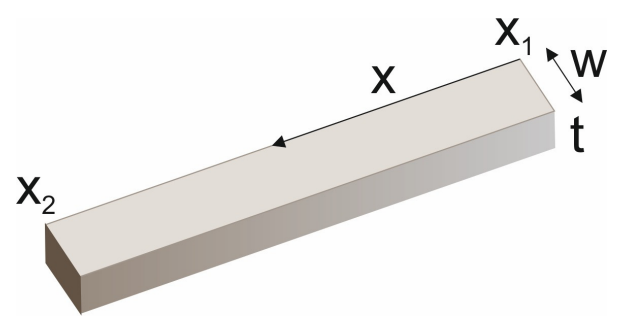

Figure 3. Three-dimensional layout of the rectangular transmission electrode.

\subsection{Pyramid-Shaped Electrode}

In order to obtain curved voltage profiles, a straightforward way from the design and fabrication point of view is by progressively increasing the electrode width towards the lens center, as depicted in Figure 4c. This can apply both in the voltage divider and the orthogonal configurations, shown in Figure $4 \mathrm{a}, \mathrm{b}$, respectively. As a consequence, a linearly decreasing resistance $R(x)$ towards the lens center is obtained leading to a curved voltage drop profile. The curvature depends on the relation between $w_{1}$ and $w_{1}^{\prime}$. In this case, the electrode width is described by the linear equation

$$
w(x)=w_{1}+\frac{w_{1}^{\prime}-w_{1}}{L} x=w_{1}+\frac{\Delta w}{L} x=w_{1}+k x,
$$

where $L$ is the electrode length. The electrode resistance $R(x)$ is calculated according to Equation (4) as follows

$$
R(x)=R_{\mathrm{sq}} \int_{0}^{x} \frac{d x}{w_{1}+k x}=\frac{R_{\mathrm{sq}}}{k} \ln \left(\frac{w_{1}+k x}{w_{1}}\right),
$$

where, without loss of generality, we have consider $x_{1}=0, x_{2}=L$. The total electrode resistance $R_{t}$ is given for $x=L$ and it equals

$$
R_{t}=\frac{R_{\mathrm{sq}}}{k} \ln \left(\frac{w_{1}^{\prime}}{w_{1}}\right)
$$

Subsequently, the voltage profile $V(x)$ for the pyramid-shaped transmission electrode is given by

$$
V(x)=V_{1}+\left(V_{c}-V_{1}\right) \frac{\ln \left(\frac{w_{1}+k x}{w_{1}}\right)}{\ln \left(\frac{w_{1}^{\prime}}{w_{1}}\right)},
$$

and it is a function of the Neperian logarithmic equation. By modifying the values $w_{1}$ and $w_{1}^{\prime}$ different voltage profile curvatures can be obtained. 

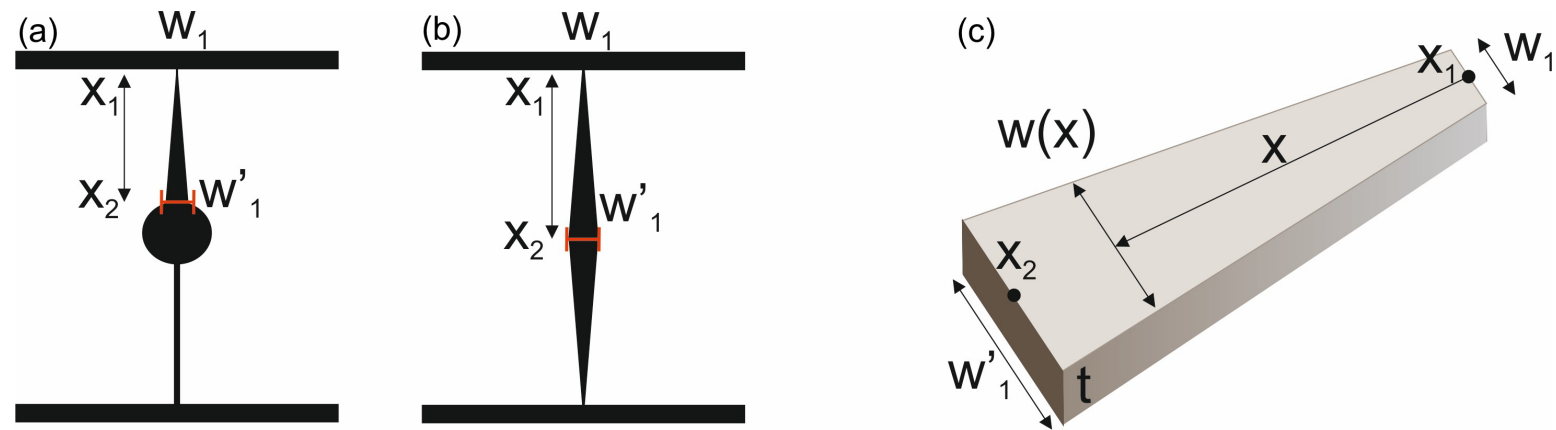

Figure 4. Depiction of the layout for a pyramid-shaped transmission electrode: (a) orthogonal and (b) voltage divider configuration. (c) Three-dimensional schematic the pyramid-shaped transmission electrode.

\subsection{Parabola-Shaped Electrode}

In order to obtain other profiles, the width of the electrode can be modified according to, in principle, any analytical equation. As a next case we study the effect of a parabola-shaped electrode. As it will be demonstrated in the results presented in Section 3, both the parabola-shaped and the pyramid-shaped electrodes can closely approximate two selected target optical phase profiles, therefore the investigation of further electrode shapes is not included in this work. Nevertheless, topologies targeting other voltage profiles can be engineered by following the same steps employed before. The proposed structure is detailed in Figure 5. The variable electrode width is given by the parabolic equation

$$
w(x)=w_{1}+\frac{w_{1}^{\prime}-w_{1}}{L^{2}} x^{2}=w_{1}+m x^{2},
$$

which equals $w_{1}$ and $w_{1}^{\prime}$ at $x=0$ and $x=L$, respectively. The resistance $R(x)$ is calculated as

$$
R(x)=R_{\mathrm{sq}} \int_{0}^{x} \frac{d x}{w_{1}+m x^{2}}=\frac{R_{\mathrm{sq}}}{\sqrt{m w_{1}}} \arctan \left(\sqrt{\frac{m}{w_{1}}} x\right),
$$

and the electrode resistance equals

$$
R_{t}=\frac{R_{\mathrm{sq}}}{\sqrt{m w_{1}}} \arctan \left(\sqrt{\frac{m}{w_{1}}} L\right)
$$

The voltage profile is then calculated as

$$
V(x)=V_{1}+\left(V_{c}-V_{1}\right) \frac{\arctan \left(\sqrt{\frac{m}{w_{1}}} x\right)}{\arctan \left(\sqrt{\frac{m}{w_{1}}} L\right)} .
$$

For the case of higher degree polynomials, sums of Neperian logarithmic equations are expected. By modifying the values $w_{1}$ and $w_{1}^{\prime}$, the voltage profile can be approximated to parabolic, hyperbolic/elliptical equations. 

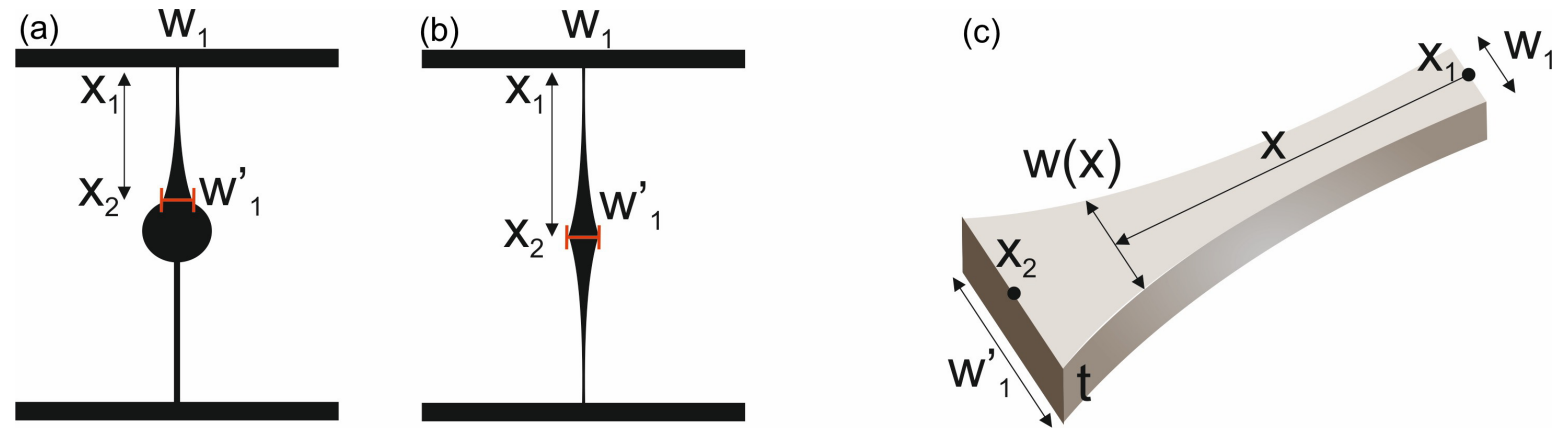

Figure 5. Depiction of the layout for a parabola-shaped transmission electrode: (a) orthogonal and (b) voltage divider configuration. (c) Three-dimensional schematic the parabola-shaped transmission electrode.

\section{Results}

\subsection{Rectangular Electrode}

The rectangular electrode is the simplest case. The voltage is proportionally distributed along the transmission electrode, as in Equation (5). In order to validate the analytical equation, we simulated the structure by using the finite-element method (FEM) implemented in the commercial tool COMSOL Multiphysics $^{\mathrm{TM}}$. For both configurations the total diameter is $1 \mathrm{~cm}$, the width of the transmission electrode is $w_{1}=w_{1}^{\prime}=10 \mu \mathrm{m}$ and the ITO resistivity is $R_{\mathrm{sq}}=100 \Omega / \mathrm{sq}$. In the case of the voltage divider, $w_{2}$ equals $60 \mu \mathrm{m}$ and the value of $V_{2}$ is selected such that $V_{c}=1 \mathrm{~V}$.

The numerical results are depicted by dots whereas the analytical results by solid lines in Figure 6. Excellent agreement is observed. By using this equation, the phase profile of an optical beam propagating through a LC lens with the calculated voltage profiles can be estimated by means of the Frank-Oseen equations. Such results can be checked in the references commented before.
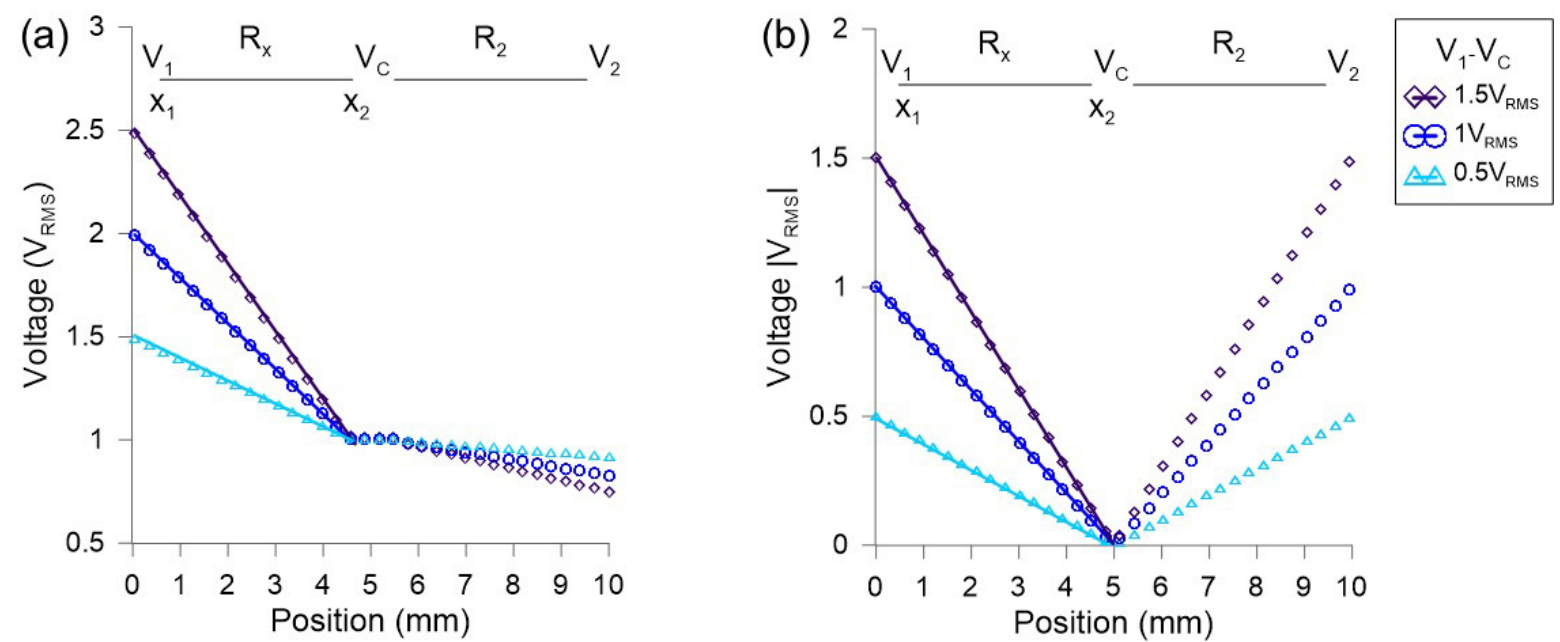

Figure 6. Simulation of the voltage distribution along the transmission line for (a) voltage divider configuration and (b) orthogonal configuration. Dots are the numerical calculations and lines the results of Equation (5). Structural parameters can be found in References [52,54].

\subsection{Pyramid-Shaped Electrode}

In this case, the voltage can be analytically estimated by Equation (9). In order to validate the analytical equation, the structure is also simulated. For both configurations the total diameter is $1 \mathrm{~cm}$, the width of the transmission electrode is $w_{1}=1 \mu \mathrm{m}$ and ITO resistivity is $R_{\mathrm{sq}}=100 \Omega / \mathrm{sq}$. For the voltage divider configuration $w_{1}^{\prime}=10 \mu \mathrm{m}$ and $w_{2}=60 \mu \mathrm{m}$, whereas for the orthogonal configuration $w_{1}^{\prime}=20 \mu \mathrm{m}$. The results are shown in Figure 7. 

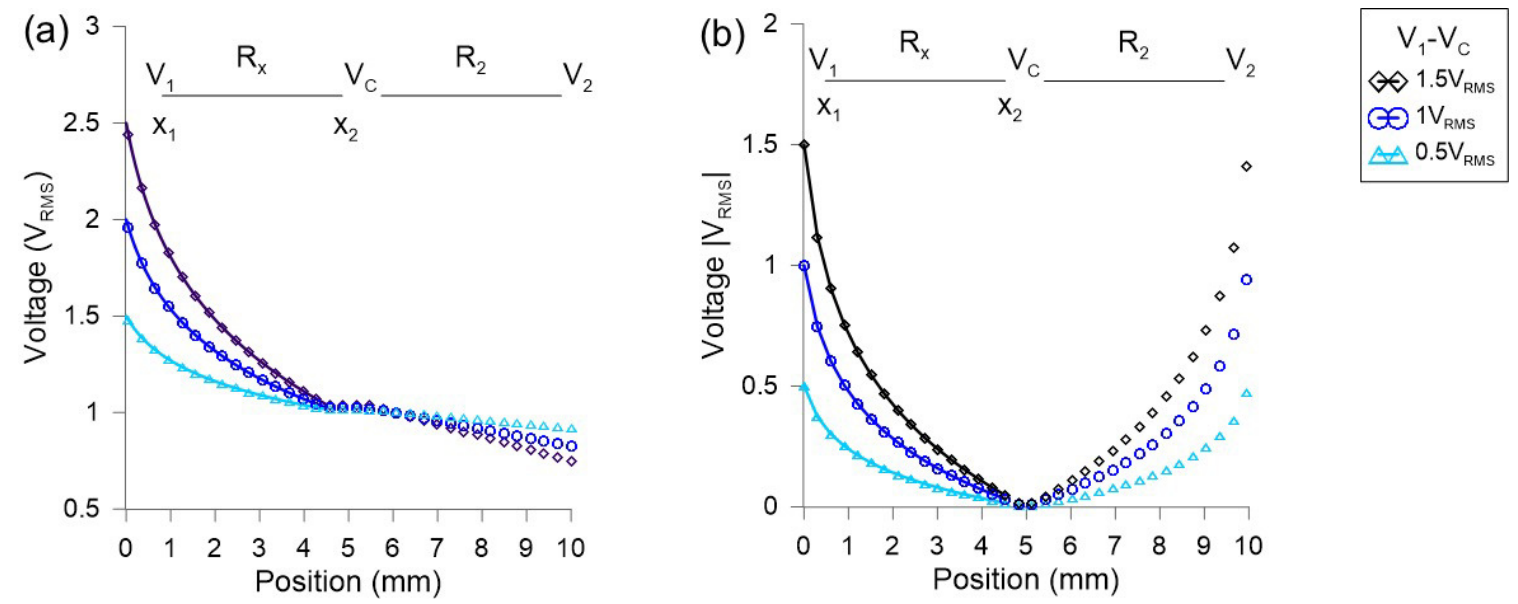

Figure 7. Simulation of the voltage distribution across the pyramid-shaped transmission line for (a) voltage divider configuration and (b) orthogonal configuration. Dots are the numerical estimations and lines the results of Equation (9). Structural parameters can be found in References [52,54].

As can be observed, as the value of $w_{1}^{\prime}$ increases the curvature also increases. The analytical equations agree with the numerical estimations validating the proposed EEC. Despite this, the results are far from optimal when the phase is compared to parabolic or hyperbolic profiles. For this reason, an optimization process is presented in Section 3.4.

\subsection{Parabola-Shaped Electrode}

In this case, the voltage can be analytical estimated by Equation (13). In order to validate the analytical equation, the structure is also simulated. In this case, only the orthogonal configuration is numerically solved to demonstrate the validity of the derived analytical equations. The used parameter are $w_{1}=1 \mu \mathrm{m}, w_{1}^{\prime}=100 \mu \mathrm{m}$ and the ITO resistivity is $R_{\mathrm{sq}}=100 \Omega / \mathrm{sq}$. The results are shown in Figure 8.

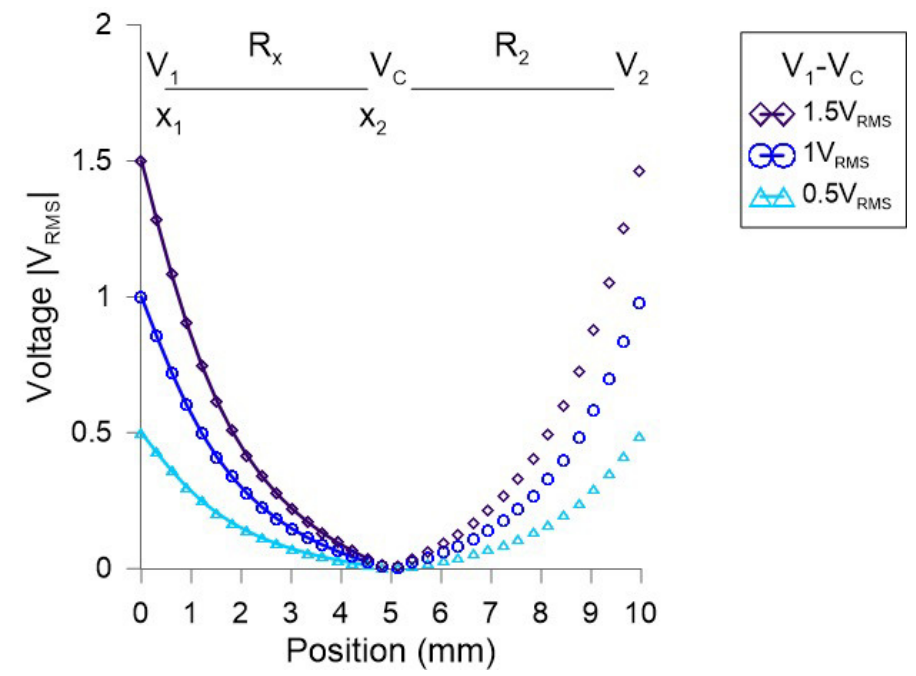

Figure 8. Simulation of the voltage distribution across the parabola-shaped transmission line for orthogonal configuration. Dots are the numerical estimations and lines the results of Equation (13).

As in the previous sections, the analytical equations agree with the numerical estimations validating the proposed EEC. We have observed that this electrode type produces a better approximation to parabolic and hyperbolic voltage profiles. Despite this, as the LC has a non-linear response this is not translated to better approximation to parabolic and hyperbolic optical phase profiles. The optimization is detailed in next section. 


\subsection{Parametric Optimization to Different Aspheric Phase Profiles}

In order to obtain some classic aspheric profiles as parabola or hyperbola, an optimization process has to be carried out. The required voltage profile for aspheric phase profiles is firstly estimated by using the Frank-Oseen Equations (LC MDA-98-1602) [57]. The key properties of the LC are reported in Table 1, where the birefringence and the permittivity are measured at $633 \mathrm{~nm}$ and $1 \mathrm{kHz}$, respectively. For the aspheric phase profiles, two unsaturated points from the effective birefringence are taken into account (from 0.05 to 0.25) as limit values, shown in Figure 9a. Then, the reference phase profiles are obtained following the canonical equations of the parabola and hyperbola, that is, the solid lines in Figure 9b. From this data, the required voltages to obtain these phase profiles, considering the Frank-Oseen equation, are estimated, shown as dashed lines in Figure 9b.

Table 1. Parameters for the nematic LC MDA-98-102.

\begin{tabular}{ccc}
\hline Elastic Constants & Birefringence & Permittivity \\
\hline$K_{11}=15.7 \mathrm{pN}$ & $n_{\mathrm{e}}=1.7779$ & $\varepsilon_{\mathrm{e}}=16.2$ \\
$K_{22}=8 \mathrm{pN}$ & $n_{\mathrm{o}}=1.5113$ & $\varepsilon_{\mathrm{o}}=12$ \\
$K_{33}=13.6 \mathrm{pN}$ & $\Delta n=0.2666$ & $\Delta \varepsilon=4.2$ \\
\hline
\end{tabular}
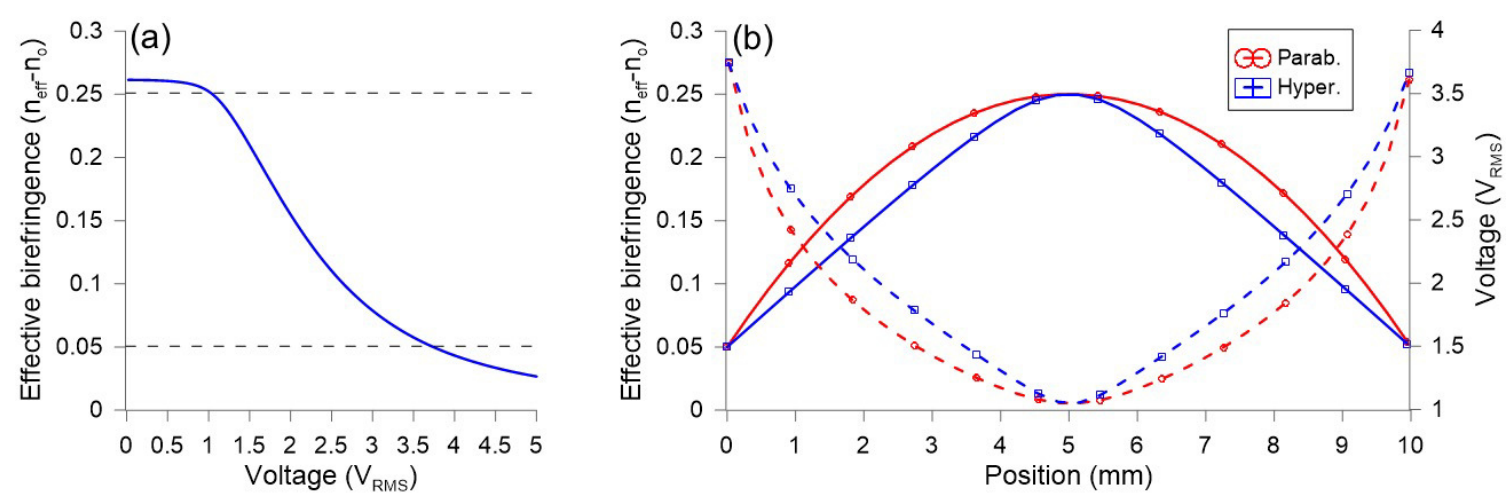

Figure 9. (a) Simulation of the effective birefringence as a function of voltage. (b) Parabolic and hyperbolic phase profiles (solid lines) and the required voltages (dashed lines) to obtain these profiles.

As can be observed, the required voltage has a non-linear curvature with respect to the phase profile caused by the non-linear voltage response of the effective birefringence. In order to determine the best manufacturing parameters the analytical expressions proposed in previous sections are adjusted to these voltage profiles. Depending on the manufacturing constraints, different parameters can be left as unknown variables. The lsqcurvefit from MATLAB is used to solve the nonlinear least-squares (nonlinear data-fitting) problem. In the following example, the square resistance and electrode length are fixed at $R_{\mathrm{sq}}=100 \Omega / \mathrm{sq}$ and $L=1 \mathrm{~cm}$, while $w_{1}$ and $w_{1}^{\prime}$ are free to vary. In fact, the important data is the relation between these two parameters, but they are free in order to allow the program to converge more easily. For this reason, the results are shown by fixing the parameter $w_{1}$ to $10 \mu \mathrm{m}$.

As can be observed in Figure 10, the pyramid-shaped electrode shows a perfect approximation to the desired aspheric profiles. Specifically, for the hyperbolic phase profile the electrode with $w_{1}=10 \mu \mathrm{m}$ and $w_{1}^{\prime}=60 \mu \mathrm{m}$ results in a $R^{2}=0.9992$. In the case of parabolic phase profile, $w_{1}=10 \mu \mathrm{m}$ and $w_{1}^{\prime}=165.8 \mu \mathrm{m}$ results in a $R^{2}=0.9993$. On the other hand, the parabola-shaped electrode results in $R^{2}=0.9911$ for the hyperbolic phase profile (with $w_{1}=10 \mu \mathrm{m}$ and $w_{1}^{\prime}=36.9 \mu \mathrm{m}$ ) and in $R^{2}=0.9908$ for the parabolic phase profile (with $w_{1}=10 \mu \mathrm{m}$ and $w_{1}^{\prime}=93 \mu \mathrm{m}$ ). The coefficient of determination $R^{2}$ reveals that pyramid-shaped electrodes produce a better approximation for these two aspherical profiles. Moreover, the design and fabrication are simpler than parabola-shaped electrode. Despite this, the latter could be useful for other custom phase profiles or even for other 
devices on which the electro-optic response is linear and more parabolic/hyperbolic voltage profiles are required.
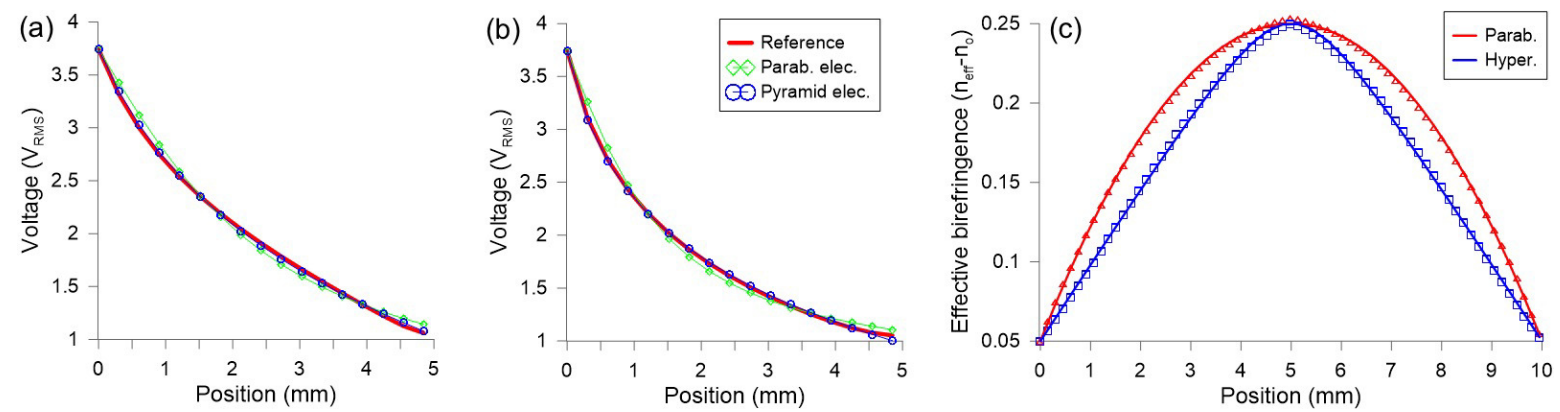

Figure 10. Results of the optimization process for (a) hyperbolic and (b) parabola phase profiles. The voltage profile of Figure $9 \mathrm{~b}$ is in a solid red line (Reference) whereas the fitted curves corresponding to parabola-shaped and pyramid-shaped electrodes are green and blue dots, respectively. (c) Parabolic (red line) and hyperbolic (blue line) phase profiles based on optimized pyramid-shaped electrodes, solid lines are the canonical equations and dots are the obtained phase profiles by using the optimized pyramid-shaped electrodes.

\subsection{Tunability of the Optimized Designs}

The phase shift modulation across the LC lens can be estimated by the optical path length $\left(d \Delta n_{\mathrm{eff}}\right)$ multiplied by $2 \pi / \lambda$, where $d$ and $\lambda$ are the LC cell thickness and the working wavelength. We define $\Delta\left(\Delta n_{\text {eff }}\right)$ as the difference between the effective birefringence in the lens center and at the lens side. In the previous section, both pyramid and parabola-shaped electrodes were optimized for obtaining a maximum phase modulation, which corresponds to $\Delta\left(\Delta n_{\text {eff }}=0.2\right)$. However, it is a known fact that LC lenses usually have different aberrated responses as the maximum phase shift modulation is modified. For this reason, a study of the tunability response of the designed electrodes is carried out in this section. The optimization process is twofold, aiming to find the best combinations of both maximum effective birefringence/corresponding voltages and structural dimensions at once to obtain the best approximation to a parabolic profile (in terms of $R^{2}$ ). For the pyramid-shaped electrode, the results (Table 2) confirm that the structural parameters of previous section behaves better for high phase shifts. There is a reduction in the fit quality as the maximum phase shift is reduced but is not considerable until $\Delta\left(\Delta n_{\text {eff }}=0.05\right)$. Therefore, the same electrode, which was optimized for $\Delta\left(\Delta n_{\text {eff }}=0.2\right)$, can perform satisfactorily for $\Delta\left(\Delta n_{\text {eff }}\right)$ values down to 0.05 , meaning that there is no need to modify the device in order to operate in different regimes of effective birefringence that produce parabolic phase profiles.

Table 2. Results of the optimization process to obtain parabolic phase profiles with pyramidshaped electrodes.

\begin{tabular}{cccccc}
\hline $\boldsymbol{\Delta}\left(\boldsymbol{\Delta} \boldsymbol{n}_{\text {eff }}\right)$ & $\boldsymbol{\Delta} \boldsymbol{n}_{\text {eff }}^{(x=0)}$ & $\boldsymbol{V}_{\mathbf{1}}$ & $\boldsymbol{\Delta} \boldsymbol{n}_{\text {eff }}^{(x=L / 2)}$ & $\boldsymbol{V}_{\boldsymbol{c}}$ & $\boldsymbol{R}^{\mathbf{2}}$ \\
\hline 0.05 & 0.1 & 2.64 & 0.15 & 2.04 & 0.9883 \\
0.1 & 0.065 & 3.3 & 0.165 & 1.9 & 0.9939 \\
0.15 & 0.07 & 3.18 & 0.22 & 1.4 & 0.9958 \\
0.2 & 0.05 & 3.74 & 0.25 & 1.04 & 0.9992 \\
\hline
\end{tabular}

On the contrary, it was found that the parabola-shaped electrode behave considerably better for larger ranges of modulation of the effective birefringence. The resulting optimized structural parameters are different from those reported in Section 3.4, as these were optimized for a range of $\Delta\left(\Delta n_{\text {eff }}\right)$ values (contrary to a sole value 0.02 in the previous Section). The optimal structural parameters are $w_{1}=10 \mu \mathrm{m}$ and $w_{1}^{\prime}=41 \mu \mathrm{m}$, which yielded the best behaviour for $\Delta\left(\Delta n_{\text {eff }}\right)=0.1$. The corresponding results are summarized in Table 3. 
Table 3. Results of the optimization process to obtain parabolic phase profiles with parabola-shaped electrodes.

\begin{tabular}{cccccc}
\hline $\boldsymbol{\Delta}\left(\boldsymbol{\Delta} \boldsymbol{n}_{\text {eff }}\right)$ & $\boldsymbol{\Delta} \boldsymbol{n}_{\text {eff }}^{(x=0)}$ & $\boldsymbol{V}_{\mathbf{1}}$ & $\boldsymbol{\Delta} \boldsymbol{n}_{\text {eff }}^{(x=L / 2)}$ & $\boldsymbol{V}_{\boldsymbol{c}}$ & $\boldsymbol{R}^{\mathbf{2}}$ \\
\hline 0.05 & 0.115 & 2.43 & 0.165 & 1.9 & 0.9991 \\
0.1 & 0.115 & 2.43 & 0.215 & 1.45 & 0.9939 \\
0.15 & 0.095 & 2.71 & 0.245 & 1.12 & 0.999 \\
0.2 & 0.05 & 3.74 & 0.25 & 1.04 & 0.9893 \\
\hline
\end{tabular}

These results reveal that parabola-shaped electrodes have a more stable response to phase shift changes (tunability). The pyramid-shaped electrodes have the best response for high phase shift modulation but a considerable reduction of the fitting quality as this phase shift is reduced. For these reasons, when tunability prevails over optical power, parabola-shaped electrodes are recommended.

\section{Conclusions}

An equivalent electric circuit and new analytical expressions based on the recently proposed transmission electrode technique have been studied and numerically demonstrated. Thanks to the modification of only one structural parameter (the width of one part of the electrode), it is possible to engineer all kinds of aspheric LC lenses (key to obtain aberration-free lenses). The results reveal that pyramid-shaped electrodes produce a better approximation for two aspherical phase profiles. In addition, this solution is easy to design and fabricate. On the other hand, the parabola-shaped electrode have a better response when tunability is required, especially for lower optical powers. In particular, the parabola-shaped electrodes can produce a broader range of parabolic voltage profiles so they can be useful in other devices with a linear electro-optic response. The optimization process yielded the optimum structural parameters and voltages to obtain almost perfect profiles with a single electrode structure. Moreover, the parabola shaped electrodes can produce a more parabolic voltage profiles so can be useful in other devices with a linear electro-optic response. For these reasons, this novel technique in combination with the proposed design equations could open new avenues of research in adaptive-focus lenses based on voltage control.

Author Contributions: J.F.A. proposed the concept and conducted part of the theoretical investigation. D.C.Z. contributed to the theoretical analysis and processing of the results. L.R.-C., J.M.S.-P. and J.M.L.-H. supervised the research. All authors participated in drafting and proof-reading the manuscript. All authors have read and agreed to the published version of the manuscript.

Funding: This work was supported by Comunidad de Madrid and FEDER Program (S2018/NMT-4326), the Ministerio de Economía y Competitividad of Spain (TEC2016-77242-C3-1-R and TEC2016-76021-C2-2-R), the FEDER/Ministerio de Ciencia, Innovación y Universidades and Agencia Estatal de Investigación (RTC2017-6321-1, PID2019-107270RB-C21 and PID2019-109072RB-C31).

Conflicts of Interest: The authors declare no conflict of interest.

\section{References}

1. Zhou, G.; Yu, H.; Chau, F.S. Microelectromechanically-driven miniature adaptive Alvarez lens. Opt. Express 2013, 21, 1226. [CrossRef]

2. Bawart, M.; Jesacher, A.; Zelger, P.; Bernet, S.; Ritsch-Marte, M. Modified Alvarez lens for high-speed focusing. Opt. Express 2017, 25, 29847. [CrossRef]

3. Zhang, D.Y.; Lien, V.; Berdichevsky, Y.; Choi, J.; Lo, Y.H. Fluidic adaptive lens with high focal length tunability. Appl. Phys. Lett. 2003, 82, 3171-3172. [CrossRef]

4. Ren, H.; Fox, D.; Anderson, P.A.; Wu, B.; Wu, S.T. Tunable-focus liquid lens controlled using a servo motor. Opt. Express 2006, 14, 8031. [CrossRef]

5. Sugiura, N.; Morita, S. Variable-focus liquid-filled optical lens. Appl. Opt. 1993, 32, 4181. [CrossRef]

6. Ren, H.; Wu, S.T. Variable-focus liquid lens by changing aperture. Appl. Phys. Lett. 2005, 86, 211107. [CrossRef]

7. Xiao, W.; Hardt, S. An adaptive liquid microlens driven by a ferrofluidic transducer. J. Micromech. Microeng. 2010, 20, 055032. [CrossRef] 
8. Cheng, H.C.; Xu, S.; Liu, Y.; Levi, S.; Wu, S.T. Adaptive mechanical-wetting lens actuated by ferrofluids. Opt. Commun. 2011, 284, 2118-2121. [CrossRef]

9. Malouin, B.A., Jr.; Vogel, M.J.; Olles, J.D.; Cheng, L.; Hirsa, A.H. Electromagnetic liquid pistons for capillarity-based pumping. Lab Chip 2011, 11, 393-397. [CrossRef]

10. Ren, H.; Wu, S.T. Adaptive Lenses Based on Soft Electroactive Materials. Appl. Sci. 2018, 8, 1085. [CrossRef]

11. Cheng, C.C.; Yeh, J.A. Dielectrically actuated liquid lens. Opt. Express 2007, 15, 7140. [CrossRef]

12. Kim, Y.; Francl, J.; Taheri, B.; West, J.L. A method for the formation of polymer walls in liquid crystal/polymer mixtures. Appl. Phys. Lett. 1998, 72, 2253-2255. [CrossRef]

13. Sato, S. Liquid-Crystal Lens-Cells with Variable Focal Length. Jpn. J. Appl. Phys. 1979, 18, 1679-1684. [CrossRef]

14. Berreman, D.W. Variable-Focus Liquid Crystal Lens System. U.S. Patent 4,190,330, 26 February 1980.

15. Hasan, N.; Banerjee, A.; Kim, H.; Mastrangelo, C.H. Tunable-focus lens for adaptive eyeglasses. Opt. Express 2017, 25, 1221-1233. [CrossRef]

16. Milton, H.E.; Morgan, P.B.; Clamp, J.H.; Gleeson, H.F. Electronic liquid crystal contact lenses for the correction of presbyopia. Opt. Express 2014, 22, 8035-8040. [CrossRef]

17. Algorri, J.F.; Bennis, N.; Urruchi, V.; Morawiak, P.; Sánchez-Pena, J.M.; Jaroszewicz, L.R. Tunable liquid crystal multifocal microlens array. Sci. Rep. 2017, 7, 17318. [CrossRef]

18. Chen, M.; He, W.; Wei, D.; Hu, C.; Shi, J.; Zhang, X.; Wang, H.; Xie, C. Depth-of-Field-Extended Plenoptic Camera Based on Tunable Multi-Focus Liquid-Crystal Microlens Array. Sensors 2020, 20, 4142. [CrossRef]

19. Algorri, J.F.; Urruchi, V.; Bennis, N.; Morawiak, P.; Sánchez-Pena, J.M.; Otón, J.M. Liquid crystal spherical microlens array with high fill factor and optical power. Opt. Express 2017, 25, 605-614. [CrossRef]

20. Algorri, J.F.; Bennis, N.; Herman, J.; Kula, P.; Urruchi, V.; Sánchez-Pena, J.M. Low aberration and fast switching microlenses based on a novel liquid crystal mixture. Opt. Express 2017, 25, 14795-14808. [CrossRef]

21. Valley, P.; Dodge, M.R.; Schwiegerling, J.; Peyman, G.; Peyghambarian, N. Nonmechanical bifocal zoom telescope. Opt. Lett. 2010, 35, 2582-2584. [CrossRef]

22. Oton, E.; Carrasco, A.; Vergaz, R.; Oton, J.M.; Sanchez-Pena, J.M.; Quintana, X.; Geday, M. 2D tunable beam steering - lens device based on high birefringence liquid crystals. In Proceedings of the 2011 International Conference on Space Optical Systems and Applications (ICSOS), Santa Monica, CA, USA, 11-13 May 2011; doi:10.1109/icsos.2011.5783671. [CrossRef]

23. Oton, E.; Morawiak, P.; Mazur, R.; Quintana, X.; Geday, M.A.; Oton, J.M.; Piecek, W. Diffractive and Refractive Liquid Crystal Devices Based on Multilayer Matrices. J. Light. Technol. 2019, 37, 2086-2093. [CrossRef]

24. Zografopoulos, D.C.; Kriezis, E.E. Switchable beam steering with zenithal bistable liquid-crystal blazed gratings. Opt. Lett. 2014, 39, 5842-5845. [CrossRef]

25. Zola, R.S.; Bisoyi, H.K.; Wang, H.; Urbas, A.M.; Bunning, T.J.; Li, Q. Dynamic Control of Light Direction Enabled by Stimuli-Responsive Liquid Crystal Gratings. Adv. Mater. 2018, 31, 1806172. [CrossRef]

26. Algorri, J.F.; Urruchi, V.; Bennis, N.; Sánchez-Pena, J.M.; Otón, J.M. Tunable liquid crystal cylindrical micro-optical array for aberration compensation. Opt. Express 2015, 23, 13899. [CrossRef]

27. Kotova, S.P.; Patlan, V.V.; Samagin, S.A.; Zayakin, O.A. Wavefront formation using modal liquid-crystal correctors. Phys. Wave Phenom. 2010, 18, 96-104. [CrossRef]

28. Davies, R.; Kasper, M. Adaptive Optics for Astronomy. Ann. Rev. Astron. Astr. 2012, 50, 305-351. [CrossRef]

29. Kao, Y.Y.; Huang, Y.P.; Yang, K.X.; Chao, P.C.P.; Tsai, C.C.; Mo, C.N. 11.1: An Auto-Stereoscopic 3D Display Using Tunable Liquid Crystal Lens Array That Mimics Effects of GRIN Lenticular Lens Array. SID Symp. Dig. Tech. Pap. 2009, 40, 111-115. [CrossRef]

30. Algorri, J.F.; del Pozo, V.U.; Sanchez-Pena, J.M.; Oton, J.M. An Autostereoscopic Device for Mobile Applications Based on a Liquid Crystal Microlens Array and an OLED Display. J. Disp. Technol. 2014, 10, 713-720. [CrossRef]

31. Algorri, J.F.; Urruchi, V.; Bennis, N.; Sanchez-Pena, J.M.; Oton, J.M. Cylindrical Liquid Crystal Microlens Array with Rotary Optical Power and Tunable Focal Length. IEEE Electron Device Lett. 2015, 36, 582-584. [CrossRef]

32. Algorri, J.F.; Urruchi, V.; Bennis, N.; Morawiak, P.; Sanchez-Pena, J.M.; Oton, J.M. Integral Imaging Capture System with Tunable Field of View Based on Liquid Crystal Microlenses. IEEE Photonics Technol. Lett. 2016, 28, 1854-1857. [CrossRef] 
33. Pinzon, P.J.; Vazquez, C.; Perez, I.; Sanchez-Pena, J.M. Synthesis of Asymmetric Flat-Top Birefringent Interleaver Based on Digital Filter Design and Genetic Algorithm. IEEE Photonics J. 2013, 5, 7100113. [CrossRef]

34. Kato, A.; Nakatsuhara, K.; Hayama, Y. Switching Operation in Tunable Add-Drop Multiplexer with Si-Grating Waveguides Featuring Ferroelectric Liquid Crystal Cladding. J. Lightwave Technol. 2014, 32, 4464-4470. [CrossRef]

35. Algorri, J.F.; Urruchi, V.; Bennis, N.; Sánchez-Pena, J.M. Modal liquid crystal microaxicon array. Opt. Lett. 2014, 39, 3476-3479. [CrossRef]

36. Algorri, J.F.; Love, G.D.; Urruchi, V. Modal liquid crystal array of optical elements. Opt. Express 2013, 21, 24809-24818. [CrossRef]

37. Algorri, J.; Urruchi, V.; García-Cámara, B.; Sánchez-Pena, J. Liquid Crystal Lensacons, Logarithmic and Linear Axicons. Materials 2014, 7, 2593-2604. [CrossRef]

38. Albero, J.; Garcia-Martinez, P.; Bennis, N.; Oton, E.; Cerrolaza, B.; Moreno, I.; Davis, J.A. Liquid Crystal Devices for the Reconfigurable Generation of Optical Vortices. J. Lightwave Technol. 2012, 30, 3055-3060. [CrossRef]

39. Algorri, J.F.; Urruchi, V.; Garcia-Camara, B.; Sanchez-Pena, J.M. Generation of Optical Vortices by an Ideal Liquid Crystal Spiral Phase Plate. IEEE Electron Device Lett. 2014, 35, 856-858. [CrossRef]

40. Caño-García, M.; Quintana, X.; Otón, J.M.; Geday, M.A. Dynamic multilevel spiral phase plate generator. Sci. Rep. 2018, 8, 15804. [CrossRef]

41. Kotova, S.P.; Mayorova, A.M.; Samagin, S.A. Formation of ring-shaped light fields with orbital angular momentum using a modal type liquid crystal spatial modulator. J. Opt. 2018, 20, 055604. [CrossRef]

42. Xu, Q.; Schmidt, B.; Pradhan, S.; Lipson, M. Micrometre-scale silicon electro-optic modulator. Nature 2005, 435, 325-327. [CrossRef]

43. Algorri, J.; Urruchi, V.; García-Cámara, B.; Sánchez-Pena, J. Liquid Crystal Microlenses for Autostereoscopic Displays. Materials 2016, 9, 36. [CrossRef]

44. Kim, S.U.; Na, J.H.; Kim, C.; Lee, S.D. Design and fabrication of liquid crystal-based lenses. Liq. Cryst. 2017, 1-12. [CrossRef]

45. Bailey, J.; Morgan, P.; Gleeson, H.; Jones, J. Switchable Liquid Crystal Contact Lenses for the Correction of Presbyopia. Crystals 2018, 8, 29. [CrossRef]

46. Algorri, J.F.; Zografopoulos, D.C.; Urruchi, V.; Sánchez-Pena, J.M. Recent Advances in Adaptive Liquid Crystal Lenses. Crystals 2019, 9, 272. [CrossRef]

47. Lin, Y.H.; Wang, Y.J.; Reshetnyak, V. Liquid crystal lenses with tunable focal length. Liq. Cryst. Rev. 2017, 5, 111-143. [CrossRef]

48. Li, L.; Bryant, D.; Bos, P.J. Liquid crystal lens with concentric electrodes and inter-electrode resistors. Liq. Cryst. Rev. 2014, 2, 130-154. doi:10.1080/21680396.2014.974697. [CrossRef]

49. Peyghambarian, N.; Li, G.; Mathine, D.; Valley, P.; Schwiegerling, J.; Honkanen, S.; Äyräs, P.; Haddock, J.N.; Malalahalli, G.; Kippelen, B. Electro-Optic Adaptive Lens as a New Eyewear. Mol. Cryst. Liq. Cryst. 2006, 454, 157-166. [CrossRef]

50. Abramochkin, E.G.; Vasiliev, A.A.; Vashurin, P.V.; Zhmurova, L.I.; Ignatov, V.A.; Naumov, A.F. Controlled liquid crystal lens. Prepr. PN Lebedev Phys. Inst. 1988, 194, 18.

51. Naumov, A.F.; Loktev, M.Y.; Guralnik, I.R.; Vdovin, G. Liquid-crystal adaptive lenses with modal control. Opt. Lett. 1998, 23, 992-994. [CrossRef]

52. Algorri, J.F.; Morawiak, P.; Bennis, N.; Zografopoulos, D.C.; Urruchi, V.; Rodríguez-Cobo, L.; Jaroszewicz, L.R.; Sánchez-Pena, J.M.; López-Higuera, J.M. Positive-negative tunable liquid crystal lenses based on a microstructured transmission line. Sci. Rep. 2020, 10, 10153. [CrossRef]

53. Algorri, J.F.; Morawiak, P.; Zografopoulos, D.C.; Bennis, N.; Spadlo, A.; Rodriguez-Cobo, L.; Jaroszewicz, L.R.; Sanchez-Pena, J.M.; Lopez-Higuera, J.M. Cylindrical and Powell liquid crystal lenses with positive-negative optical power. IEEE Photonics Technol. Lett. 2020, 32, 1057-1060. [CrossRef]

54. Algorri, J.F.; Morawiak, P.; Zografopoulos, D.C.; Bennis, N.; Spadlo, A.; Rodríguez-Cobo, L.; Jaroszewicz, L.R.; Sánchez-Pena, J.M.; López-Higuera, J.M. Multifunctional light beam control device by stimuli-responsive liquid crystal micro-grating structures. Sci. Rep. 2020, 10, 13806. [CrossRef]

55. Urruchi, V.; Algorri, J.F.; Marcos, C.; Sánchez-Pena, J.M. Note: Electrical modeling and characterization of voltage gradient in liquid crystal microlenses. Rev. Sci. Instrum. 2013, 84, 116105. [CrossRef] 
56. Algorri, J.F.; Urruchi, V.; Bennis, N.; Sanchez-Pena, J.M. Using an Analytical Model to Design Liquid Crystal Microlenses. IEEE Photonics Technol. Lett. 2014, 26, 793-796. [CrossRef]

57. Algorri, J. Modeling electro-optical response of nematic liquid crystals by numerical methods. Opt. Pura Y Apl. 2013, 46, 327-336. [CrossRef]

(c) (

(c) 2020 by the authors. Licensee MDPI, Basel, Switzerland. This article is an open access article distributed under the terms and conditions of the Creative Commons Attribution (CC BY) license (http:/ / creativecommons.org/licenses/by/4.0/). 